“ (C) 2016 IEEE. Personal use of this material is permitted. Permission from IEEE must be obtained for all other uses, in any current or future media, including

reprinting/republishing this material for advertising or promotional purposes, creating new collective works, for resale or redistribution to servers or lists, or reuse of any copyrighted component of this work in other works." 


\title{
Reconfigurable multi-slot antenna for bio-medical applications
}

\author{
Laure Huitema ${ }^{1}$, Hang Wong ${ }^{2, \#, ~ W e i ~}$ Lin $^{2}$ and Aurelian Crunteanu ${ }^{1}$ \\ ${ }^{1}$ Xlim Laboratory UMR CNRS 7252 - University of Limoges, 123, avenue Albert Thomas - 87060 LIMOGES CEDEX, \\ Email: laure.huitema@xlim.fr \\ ${ }^{2}$ Department of Electronic Engineering and State Key Laboratory of Millimeter Waves (HK), City University of Hong Kong \\ Tat Chee Avenue, Kowloon, Hong Kong, \\ Corresponding author (email): hang.wong@cityu.edu.hk
}

\begin{abstract}
We present a polarization- reconfigurable multislot antenna with four switchable linear polarizations (as $0^{\circ}, \pm$ $45^{\circ}$ and $90^{\circ}$ ) for implant communications. The design is based on four bow-tie shaped slots acting as radiators etched on a circular metallization plane with $45^{\circ}$ rotated sequential arrangements. RF switches based on PIN diodes are connected across each slot in order to modify the radiators polarization. We apply a differential source to feed the slot antenna through a groundtapered Balun. In order to obtain a broadside radiation pattern, a reflector is placed at the quarter-wave distance below the radiator. Measured results are showing that the realized multislot antenna can generate four switchable linear polarization states with wide bandwidth and stable gain. This polarization diversity feature makes the proposed antenna highly attractive for implant and body-centric wireless communication systems for minimizing the multi-path distortion and polarization mismatching in the wireless channels.
\end{abstract}

Index Terms - Linear polarization reconfigurable antenna, slot antenna, PIN diodes, body-centric communication system.

\section{INTRODUCTION}

Reconfigurable antennas have become more and more popular with the rapid development of modern wireless communication systems. Antennas with circular or linear polarization reconfigurable features have many noticeable advantages such as enhancing the communication channel capacity, avoiding multipath interference, and being capable of polarization coding [1-3]. Many studies have been conducted in the domain of polarization reconfigurable antennas, as reviewed in [4]-[8]. As most of the designs are based on modifying microstrip patch radiators, the operating bandwidths are commonly narrow. In addition, none of these designs can realize four switchable linear polarizations. Antennas with polarization diversity are very promising to be used as receiving antenna in order to compensate for polarization mismatch due to improper orientations of transmitters.

In wireless communication channels, multi-path distortion and polarization mismatching are critical concerns which may deteriorate the quality of the signal transmission. In order to improve the quality of wireless links, antennas with polarization diversity are widely used in modern wireless systems [9]. One example of related potential applications is the body-centric wireless communication system (BWCS) as

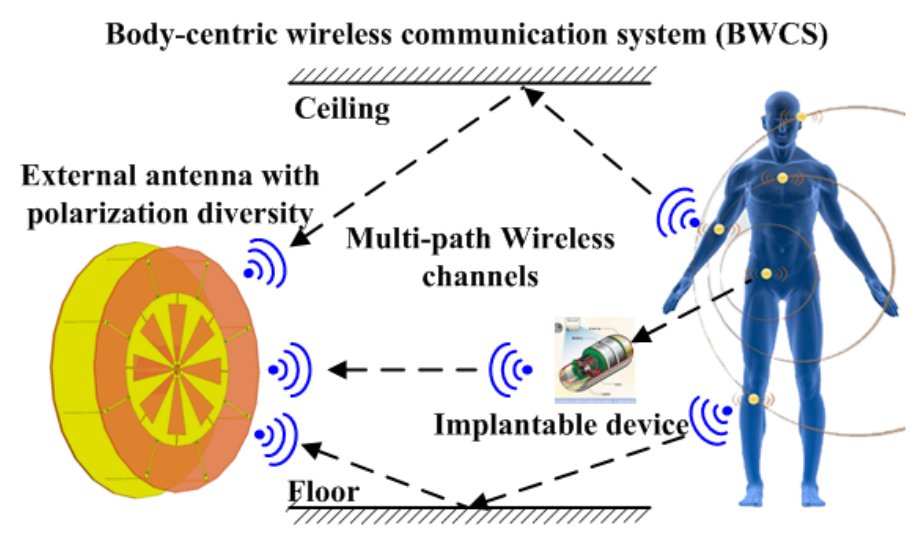

Fig. 1. Application diagram.

introduced in [10]. There are commonly two approaches in BWCS including inductive link (short range) and far-field radio-frequency link (long range) [11]. Compared with inductive link using coils, the far-field telemetry has the benefit of longer communication distances and higher data rate for information transmission. A lot of researches show that onbody, off-body and implanted wireless devices are usually operated in the linear-polarization mode which could be realized by PIFAs, meandered monopoles and short dipoles topologies. In practice, the orientation of these devices is arbitrary, due to freely movements of human body. In addition, signals send out by the on/off-body and implanted devices might create multiple-path fading as seen in Fig. 1. In this scenario, the receiving external antenna is requiring the polarization diversity feature in order to overcome the multipath distortion and polarization mismatching.

In this paper, we proposed a reconfigurable multi-slot antenna which can switch between four linear polarizations such as $0^{\circ}, \pm 45^{\circ}$ and $90^{\circ}$ at frequencies between $2.2 \mathrm{GHz}$ and $2.6 \mathrm{GHz}$. We adopted PIN diodes to switch the polarization mode of the four slot radiators. The design allows obtaining broadside radiations with wide bandwidth and stable 
gain for all modes. Our proposed antenna with the polarization diversity is capable of scanning different polarizations for minimizing the polarization mismatched and compensating the effect from multi-path distortion in wireless channels.

\section{ANTENNA DESIGN AND PERFORMANCES}

\section{A. Antenna configuration}

The proposed reconfigurable multi-slot antenna consists of three parts, as seen in Fig. 2. The multi-slot radiator is etched on the top metallization layer of a ROGERS 5880 substrate with a permittivity of 2.2 and thickness of $0.79 \mathrm{~mm}$. A reflector on the top metallization layer of a FR-4 substrate is placed below the radiator at a distance of a quarter wavelength at the center frequency. In order to provide the balanced differential feeding for the slots, a ground-tapered balun is clamped between the two substrates. In addition, DC lines with RF chokes are deployed for biasing the PIN diodes. Overall dimensions are $150 \mathrm{~mm}$ x $150 \mathrm{~mm}$ x $32 \mathrm{~mm}$.

\section{Top view}

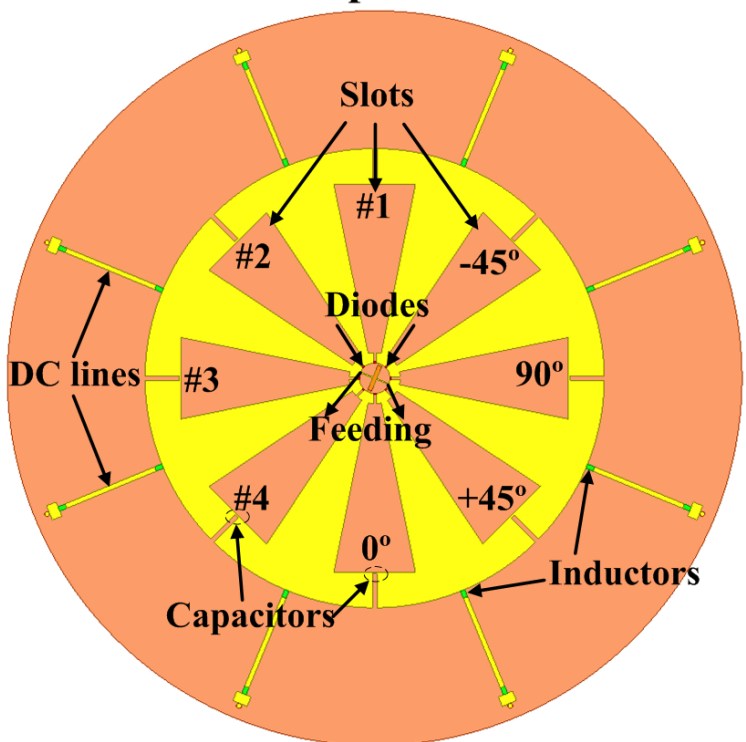

Side view

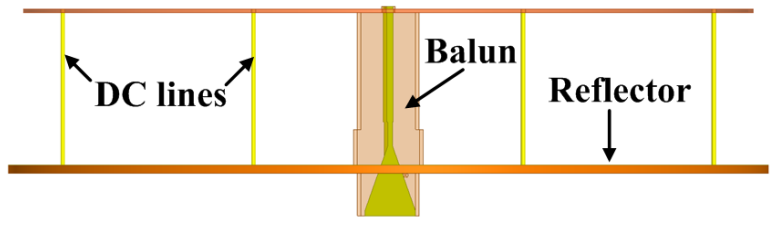

Fig. 2. Top and side view of the proposed antenna configuration.

\section{B. Switching Scheme}

We adopted PIN diodes as RF switches for adjusting the polarization states of the antenna. The implementation of the diodes connected across the slots is shown in Fig. 3. All the diodes are facing the same direction in a loop-type arrangement. When the two diodes within one pair of opposite slots are turned OFF (while the others diodes are turned ON), this specific slot pair will be in operation as the currents will flow along its contour. To control the PIN diodes, we separate the multi-slot radiator into eight parts as DC\#1 to DC\#8 by introducing capacitors as DC blocks.

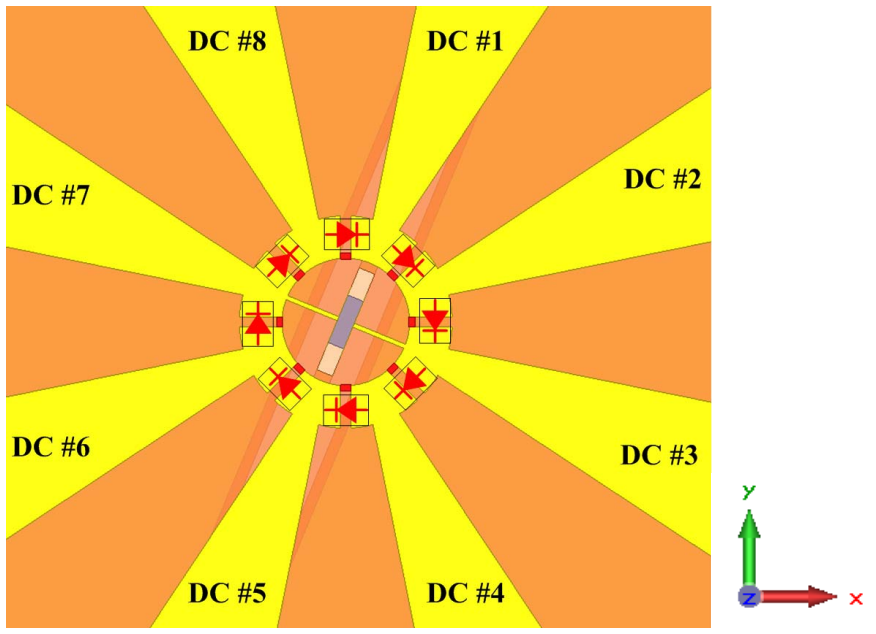

Fig. 3. PIN diodes implementation within the multi-slot design.

TABLE I

POLARIZATIONS BY DiFFERENT BIASING VOLTAGES

\begin{tabular}{|c|c|c|c|c|}
\hline \multicolumn{2}{|c|}{$3 \mathrm{~V}$} & \multicolumn{2}{|c|}{$0 \mathrm{~V}$} & Modes \\
\hline DC\#1 & $\mathrm{DC} \# 5$ & DC\#4 & $\mathrm{DC} \# 8$ & $0^{0}$ Polarization \\
\hline $\mathrm{DC} \# 4$ & $\mathrm{DC} \# 8$ & DC\#3 & $\mathrm{DC} \# 7$ & $+45^{\circ}$ Polarization \\
\hline DC\#3 & DC\#7 & $\mathrm{DC \# 2}$ & DC\#6 & $90^{\circ}$ Polarization \\
\hline $\mathrm{DC} \# 2$ & $\mathrm{DC} \# 6$ & DC\#1 & $\mathrm{DC} \# 5$ & $-45^{0}$ Polarization \\
\hline
\end{tabular}

By applying different combinations of DC biases on the switching elements, four operating polarizations can be choosen, as shown in Table I,. For the proposed antenna, only one pair of DC sources will be needed for switching to a specific antenna polarization state.

\section{Simulated results}

The simulation results of the antenna operation were obtained by using Ansoft HFSS electromagnetic simulator. In this section we present an example of a configuration $\left(0^{\circ}\right.$ polarization) and simulated main parameters of the antenna, i.e. the reflection coefficient parameters (Fig. 5) and the realized gains for each operating mode presented in Table I (Fig. 6).

Due to the symmetrical structure, the results of polarizations of $0^{\circ}$ and $-45^{\circ}$ are identical; similarly, the results for the of $45^{\circ}$ and $90^{\circ}$ polarizations are identical. The $0^{\circ}$ polarization is obtained by switching six of the eight diodes in their ON states as presented in Fig. 4. 


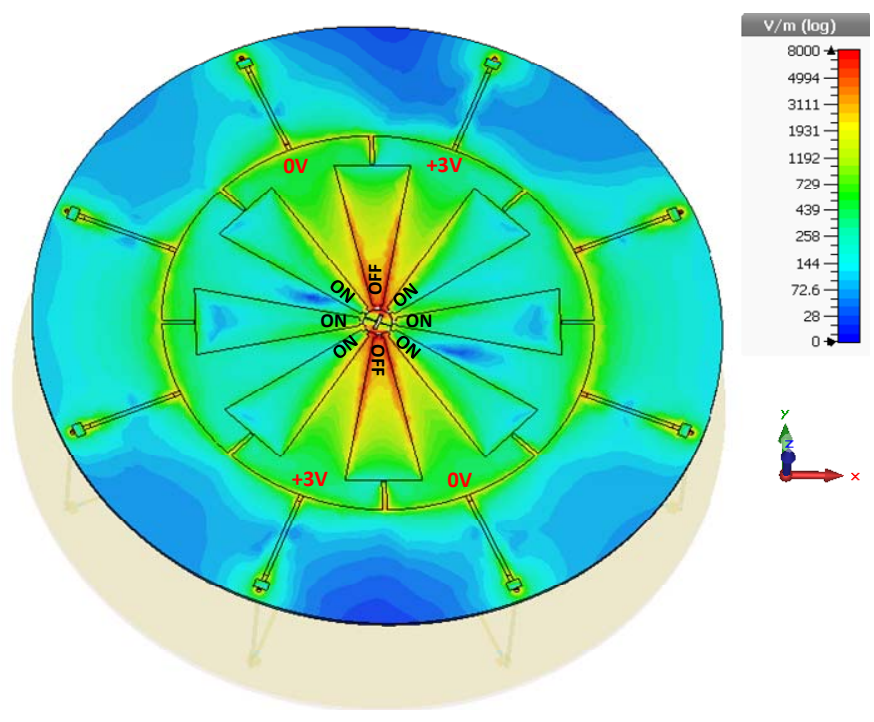

Fig. 4. E field for the $0^{\circ}$ polarization case.

A $3 \mathrm{~V}-\mathrm{DC}$ bias voltage is needed on DC\#1 and DC\#5 as mentioned on the figure and in Table I. As expected, the Efield reveals that only the slot along the y-axis is radiating: other slots are short-circuited by PIN diodes (ON states). Fig. 5 shows that the antenna is well matched across the frequency band from $2.28 \mathrm{GHz}$ to $2.64 \mathrm{GHz}$. The $-10 \mathrm{~dB}$ impedance bandwidth is $14.6 \%$.

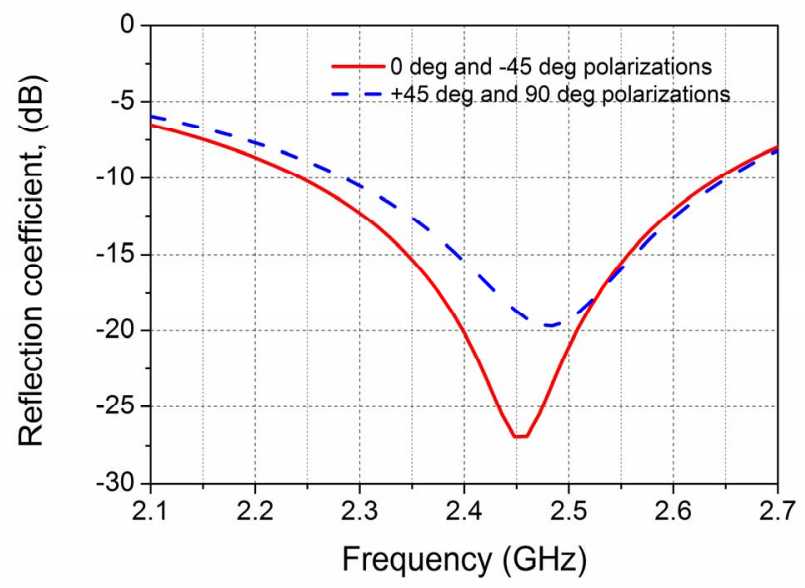

Fig. 5. Simulated reflection coefficients for the four polarisation states.

The realized gains presented in Fig. 6 show that they are stable across the operating bandwidth for all co-polarizations while having low cross polarizations. The maximum gain reaches $8 \mathrm{dBi}$.

Therefore the proposed antenna exhibits good polarization diversity with four switchable linear polarization states while having both wide bandwidths and high stable gains. The next section will present the measured results of the fabricated prototype.

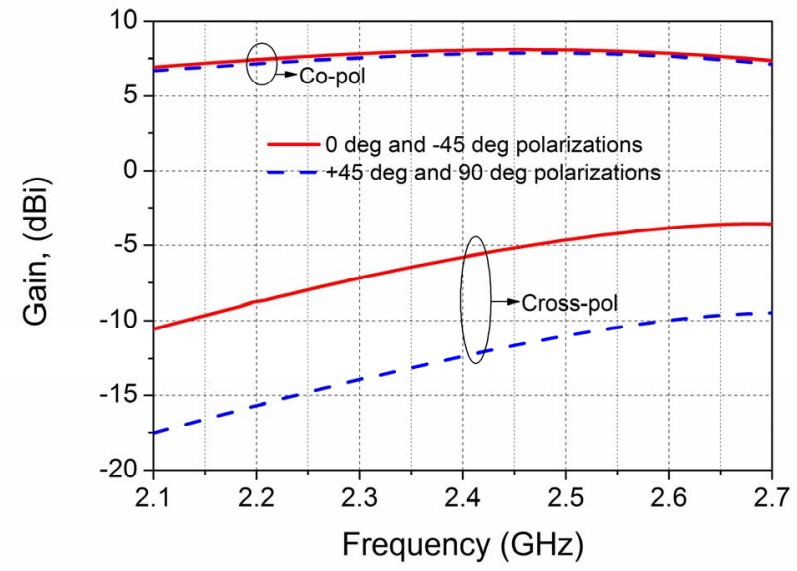

Fig. 6. Simulated gains for the four polarisation states of the antenna.

\section{Measured results}

The antenna has been fabricated and measured. The reflection coefficient was measured using an Agilent Vector Network Analyzer and the radiation patterns were acquired by a SATIMO near-field antenna measurement system.

Fig. 7 shows the measured reflection coefficient and the gain from 2.1 to $2.7 \mathrm{GHz}$. The overlapped $-10 \mathrm{~dB}$ impedance bandwidth for all radiation modes is $\sim 16 \%$ from 2.25 to 2.65 GHz. The antenna gains are stable from 2.25 to $2.6 \mathrm{GHz}$ with the maximum value of $6.7 \mathrm{dBi}$.

The radiation patterns at $2.45 \mathrm{GHz}$ for all operating modes are shown in Fig. 8. Good broadside radiation patterns are obtained with low cross polarization levels (at least less than $12 \mathrm{~dB}$ for the co-polarization). The measured results show that our proposed antenna has good radiation performance with polarization diversity and it can perform very well as a receiving antenna system for body-centric wireless applications.

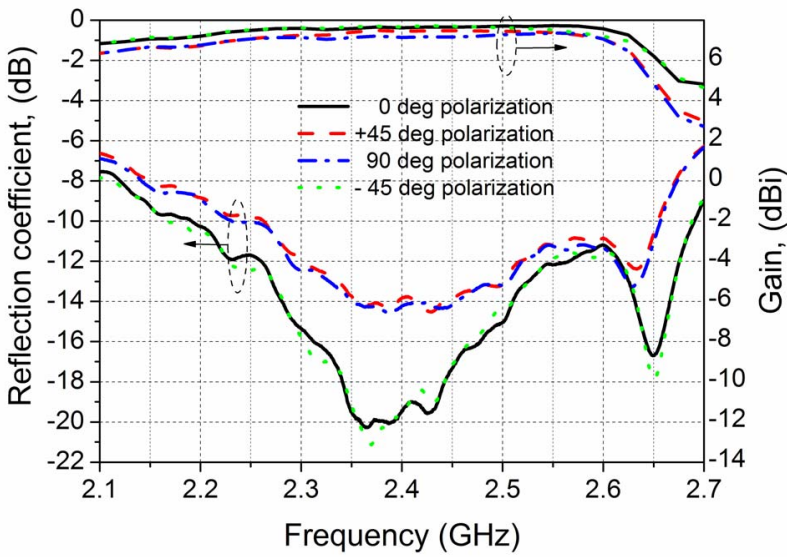

Fig. 7. Measured reflection coefficients and gains of the fabricated antenna for the four polarisation configurations. 

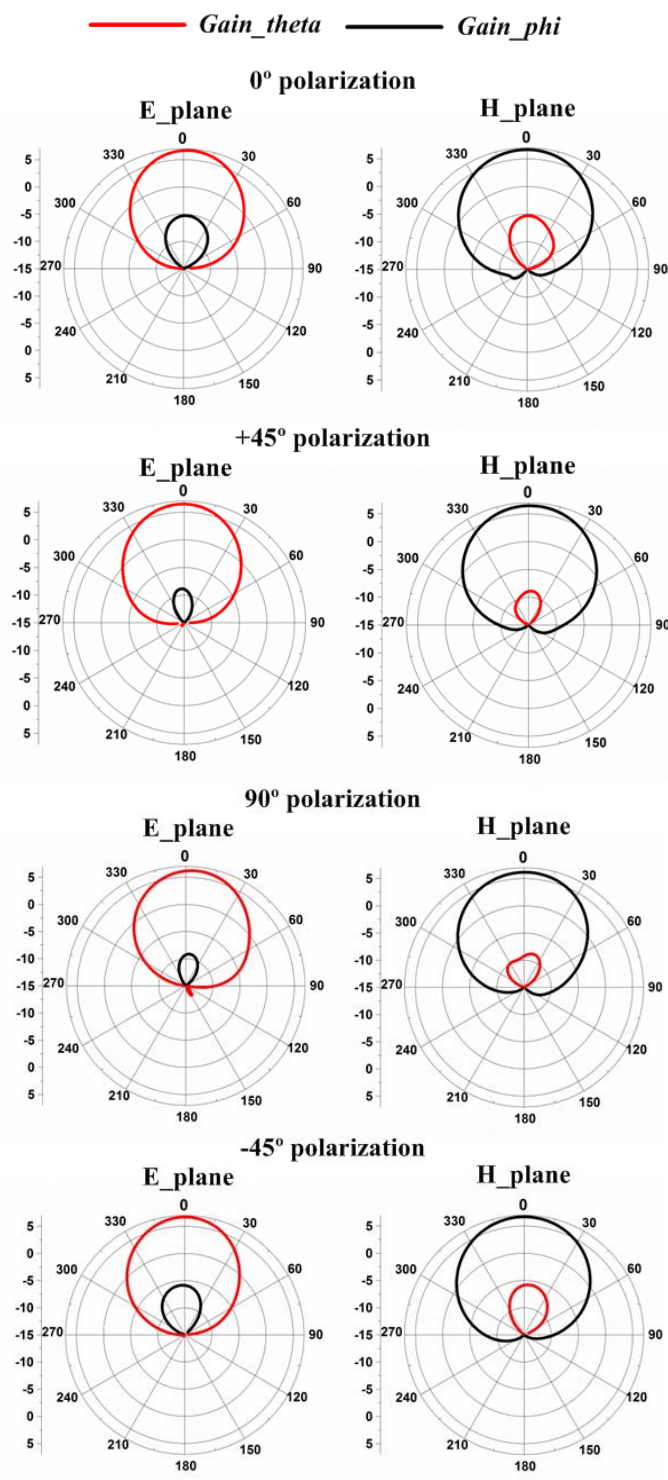

Fig. 8. Measured radiation patterns at $2.45 \mathrm{GHz}$ in two principal planes for each configurations.

\section{CONCLUSION}

Body-centric wireless communication systems need to be designed for operating in a robust way in order to compensate the influence of the surrounding medium on the implantable sensor performances (arbitrary orientation of the implanted sensors, signal multi-path fading). We presented a multi-slot antenna operating on four different linear polarizations which allows improving the quality and the reliability of wireless links between the on-body sensor and the external antenna. The polarization reconfigurability is obtained using PIN diodes, which are switching the polarization mode of the four slot radiators. A ground-tapered balun was integrated to provide a balanced differential feeding for the slots allowing a wide bandwidth and a stable gain for all modes. The measured results exhibit good polarization diversity with a realized gain up to $6.7 \mathrm{dBi}$ and low cross-polarization level.

\section{ACKNOWLEDGMENT}

This project was supported in part by the Research Grants Council of the Hong Kong SAR, China (Project No. CityU 138413) and the Fundamental Research Program of Shenzhen City under grant No. JCYJ20140509155229810.

\section{REFERENCES}

[1] M. A. Kossel, R. Kung, H. Benedickter, W. Biichtokd, "An active tagging system using circular-polarization modulation," IEEE Trans. Microw. Theory Tech., vol. 47, no. 12, pp. 2242-2248, June, 1999.

[2] W. Lin and H. Wong, "Polarization reconfigurable wheel-shaped antenna with conical-beam radiation pattern," IEEE Trans. Antennas Propag., vol. 63, no. 2, pp. 491-499, Feb, 2015.

[3] W. Lin and H. Wong, "Wideband circular polarization reconfigurable antenna," IEEE Trans. Antennas Propag., Accepted, Oct, 2015.

[4] T. Song, Y. Lee, D. Ga and J. Choi, "A polarization reconfigurable microstrip patch antenna using PIN diodes," Proceedings of APMC 2012, Dec. 4-7, 2012.

[5] P. Y. Qin, Y. Guo and C. Ding, "A dual-band polarization reconfigurable antenna for WLAN systems," IEEE Trans. Antennas Propag., vol. 61, no.11, pp. 5706-5713, Nov., 2013.

[6] M. S. Nishamol, V. P. Sarin, D. Tony, C. K. Aanandan, P. Mohanan, and K. Vasudevan, "An electronically reconfigurable microstrip antenna with switchable slots for polarization diversity," IEEE Trans. Antennas Propag., vol. 59, no. 9, pp. 3424-3427, Sep., 2011.

[7] K. Boonying, C. Phongcharoenpanich and S. Kosulvit, "Polarization reconfigurable suspended antenna using RF switches and P-I-N diodes," The 4th Joint International Conference on Information and Communication Technology, 2014.

[8] Z. X. Yang, H. C. Yang, J. S. Hong and Yang Li, "Bandwidth enhancement of a polarization-reconfigurable patch antenna with stairslots on the ground," IEEE Antenna and Wireless Propag. Letters, vol. 13 , pp. 579-582, 2014.

[9] C. B. Dietrich, K. Dietze, J. R. Nealy, W. L. Stutzman, "Spatial, polarization, and pattern diversity for wireless handheld terminals," IEEE Trans. Antennas Propag., vol. 49, no. 9, pp. 1271-1281, Sep., 2001.

[10] P. S. Hall and Y. Hao, Antennas and Propagation for Body-Centric Wireless Communications. MA: Artech House, 2006.

[11] C. R. Liu, Y. X. Guo and S. Q. Xiao," A review of implantable antennas for wireless biomedical devices," Forum for Electromagnetic Research Methods and Application Technologies (FERMAT). 